\title{
Low delay data transmission over the ATDMA air interface
}

\author{
C.Mourot, Alcatel Radiotelephone, France* \\ J.DeVile, Roke Manor Research, UK \\ E.Malkamäki, Nokia Resarch Center, Finland \\ P.Diaz, University of Catalunya, Spain \\ M.E.Gerboles, Alcatel SESA, Spain
}

\begin{abstract}
Among the features proposed for UMTS by the RACE 2084 ATDMA project is the provision for data services from 9.6 to $64 \mathrm{kbits} / \mathrm{s}$ with low transmission delay (no more than $30 \mathrm{~ms}$ ) and high integrity (bit error rate lower than $10^{-6}$ ). This paper shows how this service class can be transported over the ATDMA air interface. After a brief presentation of the latter, we develop an appropriate error control coding scheme based on code concatenation. To protect the data to the required quality while matching varying propagation and interference conditions, we define so-called transport modes. Under link adaptation control, the air interface configuration changes from one mode to another so that the target link quality can be maintained. Design parameters are dimensioned and decoding performances are assessed by means of computer and hardware simulations. We use a dedicated two-stage simulation approach based on hidden Markov modelling of the transmission channel. Results confirm the validity of the proposed scheme.
\end{abstract}

\section{INTRODUCTION}

As a candidate for UMTS (Universal Mobile Telecommunication System), the ATDMA (Advanced TDMA Mobile Access) system concept has been designed so as to support a wide range of services from voice to data or ATM-cell relay. Four testcase service classes have been defined to demonstrate the potential of the ATDMA approach: voice, low delay, long constrained delay and unconstrained delay data [1]. We concentrate here on data services with bit rate ranging from $9.6 \mathrm{kbits} / \mathrm{s}$ to $64 \mathrm{kbits} / \mathrm{s}$, low transmission delay (no more than $30 \mathrm{~ms}$ ) and high quality (bit error rate lower than $10^{-6}$ ).

After a brief description of the ATDMA air interface, we present the chosen error control scheme. This is based on classical concatenation of inner convolutional and outer Reed-Solomon codes. Overall code rate variations are obtained through outer code shortening. To protect the data to the required quality while matching varying propagation and interference conditions, we define so-called transport modes, each of these being characterized by a given rate of coding and amount of resource (i.e. number of allocated time slots per TDMA frame). Under link adaptation control, the air interface configuration changes from one mode to another so that the target link quality can be maintained. We build such modes for several service bit rates. Computer and hardware simulations using a dedicated two-stage modelling approach based on hidden Markov chains clearly depict the potential of the proposed solution.

\section{THE ATDMA AIR INTERFACE}

A detailed description of the air interface is given in [2]. This has been designed based on speech requirements and on the desire to transport a single ISDN B-channel, i.e. $64 \mathrm{kbits} / \mathrm{s}$, with low delay on a single carrier in macrocells. The resulting macrocell TDMA frame lasts $5 \mathrm{~ms}$ and comprises 18 bursts. A burst carries a payload of 66 user symbols, 10 symbols for fast signalling, and 29 training bits a priori known to the receiver. The latter are embedded in the middle of the data to minimize the channel fluctuations which the receiver has to cope with (see fig. 2.1 below).

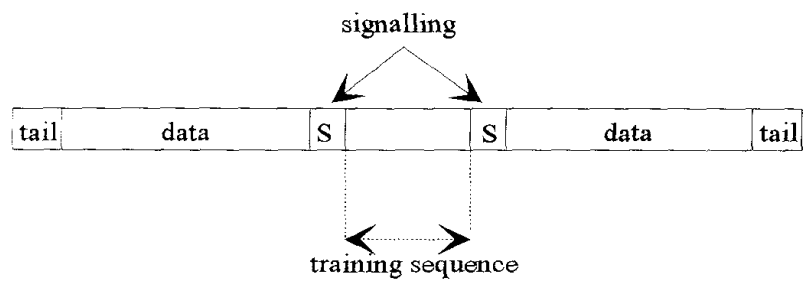

Fig.2.1. Burst structure

The macrocell carrier symbol rate is $450 \mathrm{kbauds}$. Due to relaxed propagation conditions in micro and picocells, the number of bursts per frame has been increased up to 72 and the carrier symbol rate became $1.8 \mathrm{Mbauds}$. A common modulation format of binary offset QAM is used in all cells [3]. Provision is made for switching to quaternary offset QAM to support higher data rate services or to GMSK to allow for greater cell range.

\footnotetext{
* C.Mourol is now with TELEMATE Mobile Consultants,

29/31 rue Victor Basch, 94300 Vincennes, France
} 


\section{DESCRIPTION OF ERROR CONTROL CODING SCHEMES}

Due to the tight delay constraint (not more than $30 \mathrm{~ms}$ ), no retransmission is possible and the required integrity has to be achieved by means of forward error correction only. Hence, we resort to a classical concatenated coding scheme with inner convolutional and outer Reed-Solomon (RS) code.

The overall coding rate required to achieve the integrity requirement of $10^{-6}$ in macro-cells has been estimated to be rate- $1 / 4$. Confirmation of this approximate rate is required following simulation work. However, if this is taken as a minimum code rate, then the coding rates for other cell types may be derived from this, either on a fixed basis or by puncturing this standard rate in some flexible fashion.

Inner code

The convolutional code is the rate- $1 / 2$ puncturable code of constraint length 7, given in [4]. Soft decoding will be performed by the Viterbi algorithm.

\section{Outer code}

We use the $(63 ; 48)$ code with a minimum distance $d_{\min }$ of 16 over the Galois field $\mathrm{GF}\left(2^{6}\right)$. We simply extend it by adding an overall parity check symbol to obtain a ( $64 ; 48$ ) code with $d_{\min }=17$. We then shorten it down to the appropriate dimensions for the considered service bit rate. However, when appropriate, we may use other codes over the same or some other Galois field, e.g. $G F\left(2^{8}\right)$. Decoding will be performed using the universal algorithm from [5] able to perform error-and-erasure decoding with near-minimum complexity.

\section{TRANSPORT MODES}

When the channel is good, a fixed coding redundancy may be overdimensioned whereas in bad channels, it may not be sufficient.

This leads to the definition of a number of Transport modes able to cope with varying propagation and interference conditions. A mode is characterized by a given configuration of the modulator and channel encoder and a certain amount of radio resource, able to guarantee a given quality at a given signal to noise plus interference ratio. The receiver is then dimensioned accordingly. Under link adaptation control, measurements of suitably chosen parameters are performed. They are then used to trigger air interface changes from one mode to another so that the target link quality can be maintained at the considered ratio of signal to noise plus interference, despite the varying propagation and interference conditions during the call.

First simulation results have shown that it may not be efficient to puncture the inner convolutional code. It seems that using an inner code with rate greater than one half does not allow to cope with the error patterns encountered at the output of the equalizer and produces error bursts too long to be correctly decoded by the outer code. Therefore, it has been decided to study variable rate schemes obtained by outer code rate variations while keeping a fixed inner rate of $1 / 2$. By varying the rate of the outer code through code shortening, an overall varying code rate is obtained from $1 / 2$ to $1 / 4$. Increasing the code rate will add more redundancy to the stream of information symbols, hence requiring more radio resources for transmission.

For all modes for all service bit rates, the source capture time is $10 \mathrm{~ms}$ and the modulation is assumed to be binary offset QAM. The nomenclature for the modes is given in the form cell type service class and bit rate mode number.

\section{$9.6 \mathrm{kbits} / \mathrm{s}$}

With a capture time of $10 \mathrm{~ms}$, 96 bits require transport over the air interface, within the delay budget of $30 \mathrm{~ms}$. When only a single slot per TDMA frame can be utilised, the maximum interleaving depth permitted within the delay budget is 4 . However higher data rates, achieved through employing multiple slot allocation in the TDMA frame, allows the interleaving depth (in terms of number of halfbursts) to be increased accordingly.

Mode mac ld9.6_1: we use the inner code only, unpunctured. 96 information bits +3 tail bits are encoded into 198 bits. they are then interleaved on the basis of two half-bursts in frame $n$ and one half-burst in frame $n+1$ over four successive frames. ${ }^{1}$ (see fig.4.1).

Mode mac $l d 9.62$ : the concatenated scheme is used. 96 information bits are encoded by the rate- $3 / 4$ RS $(22 ; 16)$ code. This code is obtained from the mother code $(63 ; 48)$ by dividing its dimensions by 3 . The resulting $126+6$ tail bits are encoded by the unpunctured inner code. This gives 264 bits, per coded block, interleaved on the basis of two half-bursts per TDMA frame over four successive frames.

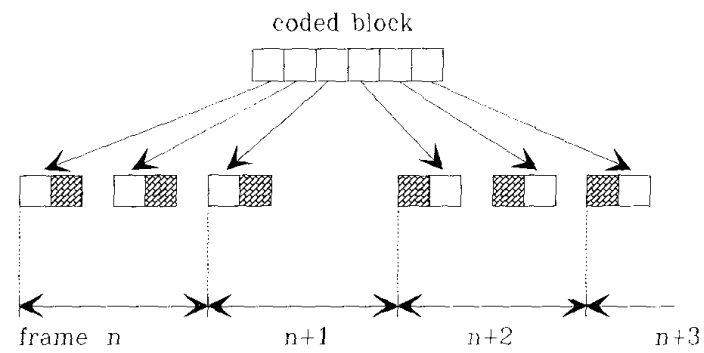

previous/subsequent block

Fig.4.1. Diagonal interleaving over 4 TDMA frames for mode mac_ld $9.6 \_1$

\footnotetext{
${ }^{1}$ Within the bursts, a symbol-interleaving mechanism similar to that used in GSM is applied.
} 
Mode mac $l d 9.63$ : Concatenated scheme. 96 information bits are encoded by the rate- $1 / 2$ RS code. The resulting $192+6$ tail bits are encoded by the inner code, unpunctured. This gives 396 bits per coded block, interleaved on the basis of three half-bursts per TDMA frame over four successive frames.

The mapping of the $9.6 \mathrm{kbits} / \mathrm{s}$ modes is summarized in table I.

TABLE I

Summary of mode characteristics for $9.6 \mathrm{kbits} / \mathrm{s}$

\begin{tabular}{|c|c|c|c|c|c|}
\hline Mode & $\begin{array}{c}\text { Inner } \\
\text { code } \\
\text { rate }\end{array}$ & $\begin{array}{c}\text { Outer } \\
\text { code } \\
\text { rate }\end{array}$ & $\begin{array}{c}\text { Gobal } \\
\text { rate }\end{array}$ & $\begin{array}{l}\text { bits per } \\
\text { coded block }\end{array}$ & $\begin{array}{c}\text { half-bursts } \\
\text { per } \\
\text { interleaving } \\
\text { period }\end{array}$ \\
\hline 1 & $1 / 2$ & 1 & $1 / 2$ & 198 & 6 \\
\hline 2 & $1 / 2$ & $3 / 4$ & $3 / 8$ & 264 & 8 \\
\hline 3 & $1 / 2$ & $1 / 2$ & $1 / 4$ & 396 & 12 \\
\hline
\end{tabular}

\section{$19.2 \mathrm{kbits} / \mathrm{s}$}

For the $19.2 \mathrm{kbits} / \mathrm{s}$ service, we use the same shortened code as for the $9.6 \mathrm{kbits} / \mathrm{s}$. A service frame now consists of 192 bits. We split it into two half-frames and apply the preceding encoding mechanism to each half-frame. For each of the above defined modes, the number of half-bursts used per TDMA frame is therefore doubled (see table II).

TABLE II

Summary of mode characteristics for $19.2 \mathrm{kbits} / \mathrm{s}$

\begin{tabular}{|c|c|c|c|c|c|}
\hline Mode & $\begin{array}{c}\text { Inner } \\
\text { code } \\
\text { rate }\end{array}$ & $\begin{array}{c}\text { Outer } \\
\text { code } \\
\text { rate }\end{array}$ & $\begin{array}{c}\text { Global } \\
\text { rate }\end{array}$ & $\begin{array}{c}\text { bits per } \\
\text { coded block }\end{array}$ & $\begin{array}{c}\text { half-bursts } \\
\text { per } \\
\text { interleaving } \\
\text { period }\end{array}$ \\
\hline 1 & $1 / 2$ & 1 & $1 / 2$ & 396 & 12 \\
\hline 2 & $1 / 2$ & $3 / 4$ & $3 / 8$ & 528 & 16 \\
\hline 3 & $1 / 2$ & $1 / 2$ & $1 / 4$ & 792 & 24 \\
\hline
\end{tabular}

\section{$64 \mathrm{kbits} / \mathrm{s}$}

Following a similar procedure as for the previous bit rates, we have identified 3 modes for this service. The first two are valid in all cells while the third one is not valid in macrocells since it uses a number of bursts per frame which cannot be provided in these cells.

mac_ld64_1: inner code only, unpunctured. 640 information bits $\mp 7$ tail bits are encoded to give 1294 bits to which we append 26 dummy bits. This is interleaved on the basis of 10 half-bursts per TDMA frame over four successive frames. mac ld64 2 : concatenated scheme. 640 information bits + 8 dummy bits are encoded by the $(54 ; 36)$ RS code obtained by shortening the $(63 ; 45)$ code over $\mathrm{GF}\left(2^{6}\right)$. The resulting 972 bits +7 tail bits are encoded by the unpunctured inner code leading to 1958 coded bits to which we append 22 dummy bits. The total coded block has then 1980 bits. This is interleaved on the basis of 15 half-bursts per TDMA frame over four successive frames.

m-pic_ld64_3: concatenated scheme. 640 information bits are encoded into 2 code words by the $(82 ; 40)$ RS code over $\mathrm{GF}\left(2^{8}\right)$. This is obtained from the $(255 ; 129)$ code "divided" by 3 . This leads to a $(85 ; 43)$ code which we shorten by 3 bits. The resulting 1312 bits +7 tail bits are encoded by the unpunctured inner code. The total coded block has then 2638 bits to which we add 2 dummy bits. This is interleaved on the basis of 20 halfbursts per TDMA frame over four successive frames.

The mapping of the $64 \mathrm{kbits} / \mathrm{s}$ modes is summarized in table III.

TABLE III

Summary of mode characteristics for $64 \mathrm{kbits} / \mathrm{s}$

\begin{tabular}{|c|c|c|c|c|c|}
\hline Mode & $\begin{array}{c}\text { Inner } \\
\text { code } \\
\text { rate }\end{array}$ & $\begin{array}{c}\text { Outer } \\
\text { code } \\
\text { rate }\end{array}$ & $\begin{array}{c}\text { Global } \\
\text { rate }\end{array}$ & $\begin{array}{c}\text { bits per } \\
\text { coded block }\end{array}$ & $\begin{array}{c}\text { half-bursts } \\
\text { per } \\
\text { interleaving } \\
\text { period }\end{array}$ \\
\hline 1 & $1 / 2$ & 1 & $1 / 2$ & 1320 & 40 \\
\hline 2 & $1 / 2$ & $2 / 3$ & $1 / 3$ & 1980 & 60 \\
\hline 3 & $1 / 2$ & $1 / 2$ & $1 / 4$ & 2640 & 80 \\
\hline
\end{tabular}

Delay budget

As can be seen from the interleaving schemes, the coded blocks are interleaved over no more than four consecutive TDMA frames. This sets the total transmission delay as $10 \mathrm{~ms}$ capture time $+15 \mathrm{~ms}$ for three frames + one time-slot duration. Hence, a little under $5 \mathrm{~ms}$ is left for processing delay to achieve the overall objective of $30 \mathrm{~ms}$.

\section{SIMULATION RESULTS}

\section{Simulation set-up}

We would like to determine the performance of the proposed error control scheme by simulation. As the target BER is $10^{-6}$, this requires to simulate a very large number of bits to generate sufficiently enough errors with reasonable confidence. The resulting workload involved in the simulation of the effect of the modulator-channeldemodulator (called hereafter the Radio Channel System, RCS, see fig.5.1) may become too large and an alternative to the simulation of the entire system has to be found. 


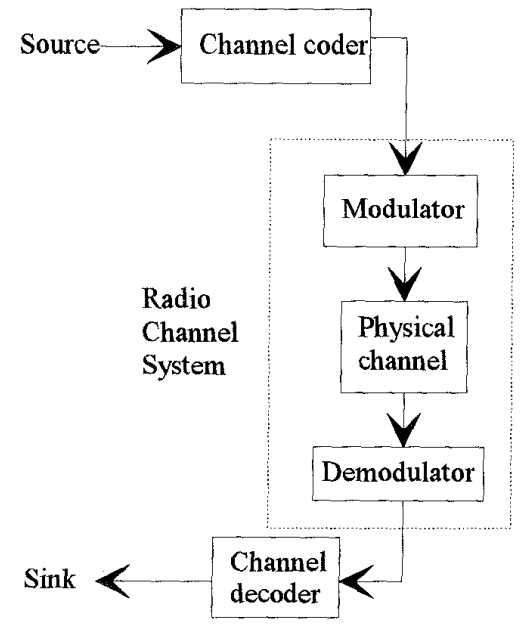

Fig.5.1. Error control coding simulation chain.

We break the simulation chain into two separate parts and replace the inner RCS by a model able to apply errors to transmitted bits as well as attaching soft information to them, just as what is seen by the channel decoder in a practical situation. If the model is accurate, the higher bit error rate of the inner system allows to run short simulations of the model while still producing sufficient accuracy. This then reduces the processing time of the whole simulation.

The model considered here consists in a two stage Markov approach. It comprises a statistical process that receives as its input the information bits and outputs the same information bits with errors and soft information according to the specific parameters of the channel that it has to model.

First, the real algorithms of the RCS are simulated separately to produce the required burst-level statistics: number of hard errors and soft decision values. Proper averaging is then performed to obtain the probability distributions of these parameters. These are used to generate the Markov model: number of states and their characteristics and the transition probabilities between states. Notice again that the number of bits to be simulated for this step is much less than required for the complete error coding simulation, since the bit error rate at the output of the equalizer is orders of magnitude higher than the one at the output of the channel decoder. Moreover, note that this also allows to compare different channel coding schemes without having to re-run the inner simulation.

In a second step, once the model is defined, a synthesis process is performed in order to generate the appropriate errors and soft decison values for the error coding simulation. The number of operations required for this last stage is very small compared to the complete simulation of the radio channel system, since it basically consists in the following steps : step 1. choose a state according to the measured probability of having a given number of hard errors in a burst.

step 2. choose the corresponding mean soft decision value according to the distribution of means attached to this state.

step 3. generate all soft decision values for this burst according to the distribution attached to the chosen mean.

Results

The BER obtained from the simulations for all modes for a $9.6 \mathrm{kbits} / \mathrm{s}$ low delay data service is shown in fig. 5.2 and 5.3. In microcells, the carrier baud rate is 1.8 Mbauds and the mobile speed is $50 \mathrm{~km} / \mathrm{h}$ whereas in macrocells, the carrier baud rate is $450 \mathrm{kbauds}$ and the mobile speed is $150 \mathrm{~km} / \mathrm{h}$. The channel model has been built by the Channel Characterization group of the ATDMA project [6]. We consider an interference limited situation, i.e. there is no additive noise. The modulation format is binary offset QAM. Perfect frequency hopping is assumed, i.e. the fades affecting consecutive bursts are uncorrelated. The inner radio channel system comprises an equalizer of the DFSE type [7] able to deliver soft outputs. 10,000 bursts have been simulated to generate the statistics for the Markov model.

To further increase the simulation speed, the algorithms have been run using the coders/decoders implemented in the project Real Time Testbed on $\mathrm{C} 40$ processors. The Markov model has also been implemented on a separate $\mathrm{C} 40$.

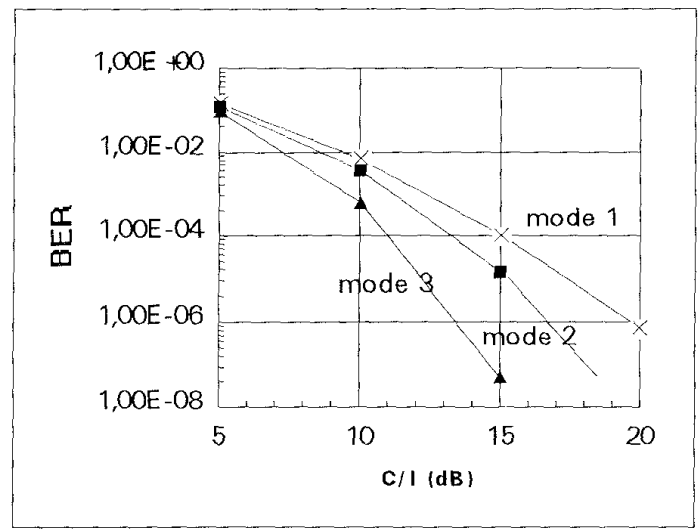

Fig.5.2. Performance of $9.6 \mathrm{kbits} / \mathrm{s}$ low delay data service Microcell, $1.8 \mathrm{Mbits} / \mathrm{s}, 40 \mathrm{~km} / \mathrm{h}$

Due to simulation complexity constraints, a scaled-down constraint length of 5 is used for the rate- $1 / 2$ puncturable convolutional code (also given in [4]), has been employed in the simulations instead of that proposed in section 3. Considering the limited available interleaving depth, no significant performance difference is expected. 
It can be seen that in microcells, the target $10^{-6} \mathrm{BER}$ is reached in mode 3 for a $C / I$ value of a little less than $14 \mathrm{~dB}$ while a BER of $10^{-4}$ is already possible at $11 \mathrm{~dB}$. Additional $3 \mathrm{~dB}$ steps are then required by lower modes. This validates the chosen range for the overall code rates. The parallel slopes of the curves below $10^{-6}$ BER for modes 1,2 and 3 indicate the validity of the proposed modes and show good decision regions for the link control functions.

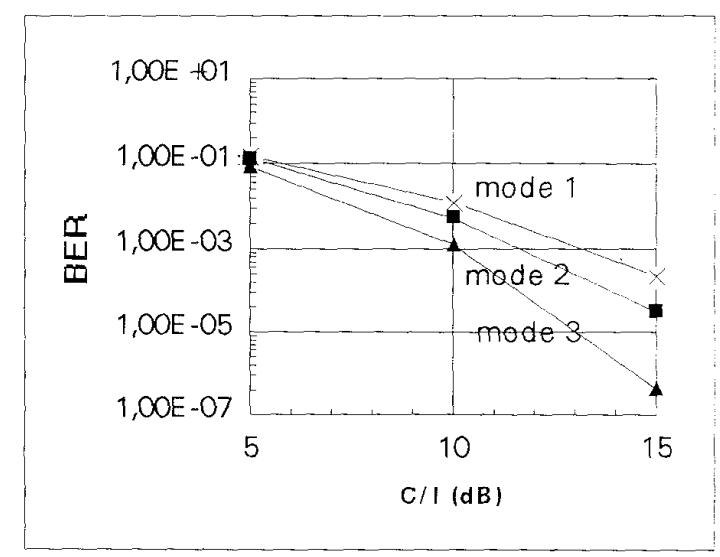

Fig.5.3. Performance of the $9.6 \mathrm{kbits} / \mathrm{s}$ transport modes, macrocell, $150 \mathrm{~km} / \mathrm{h}$.

It should be noted that due to the chosen design of transport modes for $19.2 \mathrm{kbits} / \mathrm{s}$ and the ideal frequency hopping assumption, the performance of $19.2 \mathrm{kbits} / \mathrm{s}$ modes should be exactly similar to that of the $9.6 \mathrm{kbits} / \mathrm{s}$ ones.

\section{CONCLUSION AND FUTURE WORK}

The error control coding scheme used to protect low delay data services has been presented. A classical concatenated coding scheme is used with inner convolutional and outer Reed-Solomon codes. It is characterized by a number of so-called Transport modes, each of these being determined by a given configuration of the transport chain (modulator, channel encoder, amount of resource). Five such modes have been defined for the 9.6 and $19.2 \mathrm{kbits} / \mathrm{s}$ services.

Computer and hardware simulation results show that the target performance of $10^{-6} \mathrm{BER}$ can be met by the mode comprising the highest coding rate $(1 / 4)$ at a $C / I$ value of a little less than $14 \mathrm{~dB}$ for an interleaving depth of only $20 \mathrm{~ms}$.

Future work will deal with the definition and testing of transport modes for services with bit rates of $64 \mathrm{kbits} / \mathrm{s}$ and above. Also, the validity of the two-stage modelling approach will have to be assessed for channels producing large error clusters within a transmission burst.

\section{ACKNOWLEDGMENTS}

This work has been performed within the R2084 ATDMA project to which the following organizations participate: Siemens AG, Alcatel Mobile Communication France, Alcatel SESA, Roke Manor Research, Nokia, University of Catalunya, Telecom'Paris, France Telecom CNET, DeTeMobil, Bundespost FIT, Elecktronik System Gesellschaft, Fundazione Hugo Bordoni and University of Strathclyde. In particular, the authors would like to acknowledge the contributions of their colleagues in the Transport Workpackage.

\section{REFERENCES}

[1] C.Evci, D.Florack "ATDMA testcase services" Proceedings of the RACE Mobile Telecom. Workshop, Metz, France, June 1993.

[2] J.DeVile, C.Mourot 'Determination of ATDMA air interface parameters' Proceedings of the RACE Mobile Telecom. Workshop, Metz, France, June 93.

[3] E.Malkamäki "Binary and multi-level offset QAM spectrum efficient modulation schemes for personal communications" Proceedings of the 43rd IEEE Veh. Tech. Conf., Denver, CO, May 1993.

[4] Y.Yasuda, K.Kashiki, Y.Hirate "High rate punctured convolutional codes for soft decision Viterbi decoding", IEEE Trans. Comm., Vol.32, nº3, March 1984.

[5] R.E.Blahut, "A universal Reed-Solomon decoder", IBM J. Res. Develop., Vol. 28, n⿳20, March 1984, pp150-157.

[6] J.P.Barbot et al. "Channel modelling for advanced TDMA mobile access" Proceedings of the RACE Mobile Telecom. Workshop, Amsterdam, Netherlands, May 1994.

[7] C.Mourot "A high bit rate transmission technique in mobile radio cellular environment" Proceedings of the 43rd IEEE Veh. Tech. Conf., Denver, CO, May 1993. 